\title{
LOS DIARIOS Y NOTAS DE LOS VIAJES PEDAGÓGICOS DE ROSA SENSAT VILÀ DURANTE EL PERIODO 1908-1913: ANÁLISIS CRÍTICO Y CONCEPTUAL, APORTACIONES Y DEBATES
}

\author{
The personal diaries and the notes of the pedagogical travels \\ of Rosa Sensat Vilà during the period 1908-1913: \\ critical and conceptual analysis, contributions \\ and debates
}

\section{Joan Soler Mata ${ }^{\alpha}$}

Fecha de recepción: 19/11/2018 • Fecha de aceptación: 08/02/2018

Resumen. El artículo está centrado en el análisis crítico y conceptual de los diarios y notas de los viajes que, con carácter pedagógico y de estudio, la maestra Rosa Sensat Vilà (1873-1961) realizó durante el periodo 19081913 por distintos países europeos. Nos preguntamos acerca de las personas y los lugares, las experiencias personales, las experiencias relevantes y las opiniones y valoraciones críticas plasmadas en los diarios con el objetivo de reconstruir una parte del proceso de elaboración del universo conceptual de Rosa Sensat.

Las fuentes documentales analizadas, de carácter primario y escrito, están localizadas en el Fondo histórico de la Biblioteca Rosa Sensat en Barcelona (Fondo Rosa Sensat) y en el Archivo Edad de Plata (Expedientes JAE). La metodología cualitativa utilizada es de carácter hermenéutico y conceptual, atendiendo a algunas aportaciones de la investigación biográfico-narrativa. A partir de la lectura de los diarios y notas de viaje, localizamos y sistematizamos los datos y la información disponible con el objetivo de establecer un catálogo de las instituciones visitadas y las relaciones establecidas con maestros y pedagogos europeos; y

\footnotetext{
^ Departamento de Pedagogía. Facultad de Educación, Traducción y Ciencias Humanas. Universidad de Vic - Universidad Central de Catalunya. c/ Sagrada Familia, 7.08500 Vic. España. joan.soler@ uvic.cat
} 
también para interpretar los textos y las descripciones para analizar las ideas y, sobretodo, los conceptos que incorpora a su propio ideario pedagógico.

Palabras clave: Diario personal; Viaje pedagógico; Análisis conceptual; Rosa Sensat; Escuela Nueva.

Abstract. The article is focused on the critical and conceptual analysis of the personal diaries and the travel notes, with pedagogical character, that the teacher Rosa Sensat Vila (1873-1961) made during the period 1908-1913 in different European countries. We ask ourselves about the people and the places, about the personal and relevant experiences and the opinions and the critical reviews in personal diaries in order to rebuild a part of the process of elaboration of the conceptual universe of Rosa Sensat.

The documentary sources are a primary and written nature. They are located in the Fons Historic of the Rosa Sensat Library in Barcelona and in the Archivo Edad de Plata (Fondo JAE). The qualitative methodology is a hermeneutic and conceptual nature, taking into account some contributions of biographical-narrative research. From Reading of personal diaries and travel notes, we locate and systematize the data and available information with the aim of establishing a catalogue of the institutions and the relationships with European teachers; and also to interpret the texts and the descriptions to analyze the ideas and, above all, the concepts that it incorporates to its own pedagogical ideology.

Keywords: Personal diary; Pedagogical travel; Conceptual analysis; Rosa Sensat; New Education.

\section{INTRODUCCIÓN}

El artículo, fruto de la comunicación presentada en el XIX Coloquio de Historia de la Educación celebrado en El Escorial los días 19 al 22 de septiembre de 2017, está centrado en un doble análisis crítico y conceptual de los diarios personales y notas de los viajes que, con carácter pedagógico y de estudio, la maestra Rosa Sensat Vilà (1873-1961) realizó durante el periodo 1908-1913 por distintos países europeos.

La actividad pedagógica analizada, qua ampliaremos más adelante, corresponde a un periodo de transición entre la etapa de formación y el inicio y la consolidación de su itinerario profesional. Cabe recordar que a partir de 1908 ocupó una plaza de maestra en la escuela pública de la avenida Diagonal de Barcelona y que en 1914 inició su etapa 
como directora de la Escola de Bosc del Parque de Montjuic en la misma ciudad.

\section{Objetivos}

En la investigación y el análisis realizados nos preguntamos acerca de las personas y los lugares, las experiencias personales y relevantes y las opiniones y valoraciones críticas plasmadas en los diarios con el objetivo de reconstruir una parte del proceso de elaboración del universo conceptual de Rosa Sensat. La lógica pasado-presente-futuro orienta nuestro análisis ya que intentamos partir de las experiencias previas de la maestra para descubrir cómo se incorporan a su ideario nuevos conceptos que, a la postre, conformarán un discurso pedagógico completo que se concretará en la obra educativa posterior.

Los objetivos planteados son:

$1^{\circ}$ Establecer un catálogo de las instituciones visitadas y las relaciones establecidas con maestros y pedagogos europeos.

$2^{\circ}$ Interpretar los textos y las descripciones para localizar y analizar las ideas y, sobretodo, los conceptos que incorpora a su propio ideario pedagógico.

En definitiva, más allá de una cronología y narración de los hechos acaecidos y de las prácticas e instituciones observadas, nos interesan las ideas, los conceptos y los discursos. En síntesis, se trata de rastrear los conceptos arañados o construidos durante estos viajes, en el discurso pedagógico de Rosa Sensat.

El desarrollo del artículo, después de la presentación y formulación de los objetivos, contiene los siguientes apartados: En primer lugar, se expondrá sucintamente la metodología seguida en la investigación, tanto para concretar las fuentes documentales utilizadas como para justificar los métodos cualitativos que se han elegido y, precisar finalmente, el esquema de análisis que ha servido para orientar la investigación. Después de situar los viajes pedagógicos estudiados en el contexto del itinerario formativo y profesional de Rosa Sensat, se analizarán con detalle los textos que corresponden a su estancia en Ginebra (Suiza). En la parte final se expondrán las conclusiones para dar respuesta a los objetivos planteados. 


\section{METODOLOGÍA}

Las fuentes documentales utilizadas, de carácter primario y escrito, están localizadas en el Fondo histórico de la Biblioteca Rosa Sensat en Barcelona (Fondo Rosa Sensat) ${ }^{1}$ y en el Archivo Edad de Plata (Expedientes JAE). ${ }^{2}$ En el primer caso se trata de los cuadernos que contienen los diarios y notas manuscritas de los viajes mencionados que pueden contrastarse con otros documentos como el borrador de la memoria del viaje de estudios de 1912-1913, redactada en el año 1913, con el título Métodos para la enseñanza de las ciencias físico-químicas y naturales en la escuela primaria ${ }^{3}$ (cuya versión definitiva no puede localizarse) o, también, la publicación del Ayuntamiento de Barcelona que describe y analiza el viaje de estudios de 1911. ${ }^{4}$ Del expediente de Rosa Sensat Vilà del fondo de la JAE cabe indicar que contiene algunos documentos de interés para contrastar datos, pero no conserva la memoria completa y definitiva reseñada anteriormente, aunque haya constancia de la entrega de la misma.

\footnotetext{
1 El Fondo Rosa Sensat contiene algunos documentos digitalizados, entre los cuáles los diarios y notas de los viajes pedagógicos. Disponible en: http://www2.rosasensat.org/es/pagina/ fons-historic (consultado el 3-9-2018). Estos documentos se encuentran también disponibles en el repositorio de la Memòria digital de Catalunya: http://mdc.cbuc.cat/cdm/search/collection/arxiurs.
}

2 Se trata del expediente de la maestra Rosa Sensat Vilà, pensionada por la Junta para la Ampliación de Estudios, que puede consultarse en: http://archivojae.edaddeplata.org (consultado el 3-92018).

${ }^{3}$ Rosa Sensat, Borrador de la memoria Métodos para la enseñanza de las ciencias físico-naturales en la escuela primaria (Viaje 1912-1913). Fondo Rosa Sensat. Archivo Histórico de la Biblioteca Rosa Sensat de Barcelona. Disponible en: http://www2.rosasensat.org/es/pagina/fons-historic (consultado el 3-9-2018). Este borrador, con el subtítulo «Memoria de los estudios realizados en las escuelas de Bélgica, Suiza y Alemania por Rosa Sensat», está estructurado en un índice con distintos apartados: BÉLGICA. Escuela primaria de Bélgica; Principios educativos; Las ciencias físico-químicas y naturales. Estudio del programa y su interpretación en la práctica; Secciones en diferentes escuelas primarias de la Villa; Excursiones; Las Escuelas Normales y la enseñanza de las ciencias físico-químicas y naturales; Las prácticas de enseñanza y las lecciones en su escuela de aplicación; La Escuela del Dr. Decroly; Laboratorios y museos; Material; Bibliografía. SUIZA. Escuelas primarias; El Instituto Rousseau; La Escuela Normal de Lausanne; Libros y material; El Museo del cantón de Vaud; Las enseñanzas secundarias y menagère; La escuela secundaria de Neuchâtel; La historia natural; Las escuelas de Zurich; El Museo Pestalozzi. Alemania. Las escuelas de Münich; Estrasburgo (en aquel momento bajo soberanía alemana).

${ }_{4}^{4}$ Celestina Vigneaux et al., Viaje pedagógico a Francia, Suiza y Alemania en 1911. Memoria presentada al Ayuntamiento de Barcelona por varios Maestros públicos de dicha ciudad (Barcelona: J. Horta impresor, 1912). 
La relación completa de textos analizados es la siguiente:

- Notas del viaje pedagógico de 1908 (cuaderno manuscrito).

- Viaje pedagógico a Francia, Suiza y Alemania en 1911 (publicación de 1912).

- Notas y diario del viaje de 1911 (cuadernos manuscritos).

- Notas y diario del viaje de 1912-1913 (cuatro volúmenes manuscritos).

- Memoria-borrador del viaje de 1912-1913: Métodos para la enseñanza de las ciencias físico-naturales en la escuela primaria (28 cuadernos manuscritos).

La metodología cualitativa utilizada es de carácter hermenéutico y conceptual, atendiendo a algunas aportaciones de la investigación biográfico-narrativa. Este planteamiento nos permite abordar un análisis temático a partir de la siguiente secuencia: comprender las personas (sus ideas); comprender el contexto; comprender las otras personas que interactúan, o sea, el conjunto del movimiento renovador. A partir de la lectura de los diarios y notas de viaje, hemos localizado y sistematizado los datos y la información disponible siguiendo una categorización realizada a partir de un esquema de análisis que expondremos más adelante.

El material documental estudiado pertenece sin duda a la categoría de diario establecida por Antonio Viñao:

una sucesión de textos más o menos extensos — desde la nota o apunte suelto a varios folios-, escritos sobre la marcha, al hilo de los acontecimientos, con mayor o menor frecuencia y regularidad, a lo largo de los años o durante un periodo de tiempo determinado. ${ }^{5}$

También encaja con la definición formulada por Antonio Bolívar, Jesús Domingo y Manuel Fernández, según la cual:

el diario es un registro reflexivo de experiencias (personales y profesionales) y de observaciones a lo largo de un periodo de tiempo.

\footnotetext{
${ }^{5}$ Antonio Viñao, «Las autobiografías, memorias y diarios como fuente histórico-educativa: tipología y usos», Sarmiento. Anuario Galego de Historia da Educación 3 (1999): 233.
} 
Incluye, a la vez, opiniones, sentimientos, interpretaciones, etc. Pueden adoptar un formato preferentemente descriptivo, etnográfico, analítico, evaluativo o reflexivo. Lo mejor es, quizás, que tengan una adecuada mezcla de todos. ${ }^{6}$

Los diarios y notas de viaje de Rosa Sensat tienen un carácter biográfico, pero incorporan elementos descriptivos y también juicios, opiniones y, por lo tanto, elementos de valoración e incluso propuestas y expectativas de cambio. En nuestro caso, nos centraremos en el estudio de los diarios y notas de los tres viajes mencionados y utilizaremos los demás documentos para contrastar y triangular datos y opiniones y realizar un análisis crítico. En el caso del viaje de estudios de 1912-1913, el análisis reviste complejidad e interés ya que, por una parte, contamos con los cuatro cuadernos del diario completo y la narración directa y exhaustiva del viaje en sus distintas etapas. Por otra parte, como ya hemos mencionado, contamos con la oportunidad de contrastar esta escritura más directa del diario con el borrador de la memoria dirigida a la Junta para la Ampliación de Estudios (JAE) y, por lo tanto, con una narración filtrada por el tiempo, pero también por la visión crítica de la autora. ${ }^{7}$ Ello nos permite profundizar en el análisis crítico y hermenéutico de los textos para fundamentar el análisis conceptual. ${ }^{8}$ En definitiva, en los textos podemos rastrear los conceptos fundamentales de la experiencia pedagógica de Rosa Sensat en sus viajes: los conceptos previos que constituyen su bagaje; los conceptos aprendidos y, finalmente, los conceptos elaborados. Por citar únicamente dos ejemplos, el análisis realizado nos permite descubrir las razones de la influencia de Decroly en Rosa Sensat a partir de su experiencia y visión crítica, fruto de las visitas realizadas en Bruselas; pero también nos muestra los parámetros que, a su juicio, otorgan una mejor valoración de la Escuela Normal de Lausana por encima del Instituto Rousseau de Ginebra. Ello es debido, sin duda, a las propias expectativas de la maestra, relacionadas con el interés por la

\footnotetext{
6 Antonio Bolívar, Jesús Domingo y Manuel Fernández, La investigación biográfico-narrativa en educación. Enfoque y metodología (Madrid: La Muralla, 2001), 183.

7 Véase: Paul Ricoeur, La memoria, la historia y el olvido (Madrid: Trotta, 2003).

8 Sobre este tema pueden consultarse: Faustino Oncina Coves, «Historia conceptual y hermenéutica», Azafea. Revista de Filosofía 5 (2003): 161-190; Faustino Oncina Coves (eds.), Palabras, conceptos, ideas. Estudios sobre la historia conceptual (Barcelona: Herder, 2010); Conrad Vilanou, «Historia conceptual e Historia de la educación», Historia de la Educación 25 (2006): 35-70.
} 
enseñanza y la didáctica de las ciencias físico-químicas y naturales, más que por los fundamentos psicológicos y pedagógicos de la educación. Aunque como veremos más adelante, este eje temático constituye uno de los núcleos de sus reflexiones posteriores.

En síntesis, podemos decir que la metodología utilizada se alimenta del impulso de dos motores que actúan de forma complementaria: el análisis conceptual y la investigación biográfico-narrativa.

\section{Un esquema de análisis}

En un trabajo anterior realizamos un análisis conceptual, con perspectiva histórica, de la renovación pedagógica en Catalunya. ${ }^{9}$ Dado que el universo conceptual del ideario pedagógico de Rosa Sensat debe situarse en el contexto de la renovación pedagógica del primer tercio del siglo XX, hemos decidido, sobre la base del estudio anterior, analizar los conceptos e ideas pedagógicas renovadoras de la autora, asociándolas y sistematizándolas en tres niveles: el contexto externo (social, político y cultural); el contexto institucional (modelo escolar, organización, etc.) y, en tercer lugar, el programa pedagógico (prácticas, metodologías didácticas, etc.).

\section{Gráfico 1. Un esquema de análisis}

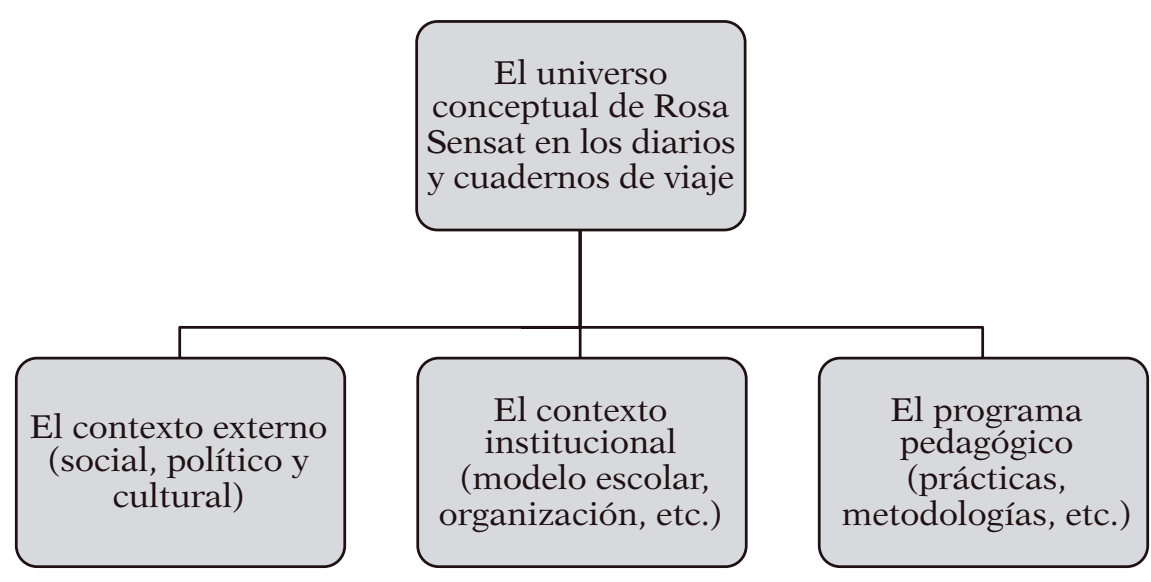

Fuente: elaboración propia.

\footnotetext{
9 Conrad Vilanou y Joan Soler, «La Historia de la Educación en Cataluña: tradición liberal e historia conceptual», Social and Education History 2, no. 3 (2013): 261-295. URL: http://dialnet.unirioja.es/ descarga/articulo/4686204.pdf (consultado el 9-6-2018).
} 
La aplicación de este esquema de análisis nos permite sistematizar la información que recogemos a partir de la lectura de los textos de los diarios o documentos del viaje. En los cuadros siguientes se exponen y visualizan dos tipos de informaciones relacionadas con el diario del primer viaje de estudios realizado en 1908. En el cuadro 1 se sistematiza la información procedente de la lectura del texto. En el cuadro 2 se sintetiza el contenido anterior y se conceptualiza la información.

Cuadro 1. Un ejemplo de sistematización a través de la lectura

\begin{tabular}{|c|c|c|c|c|}
\hline $\begin{array}{c}\text { Día/ Mes/ Año } \\
\text { - Lugar } \\
\text { - Personas e } \\
\text { instituciones }\end{array}$ & $\begin{array}{c}\text { Elementos del } \\
\text { contexto } \\
\text { externo }\end{array}$ & $\begin{array}{c}\text { Contexto } \\
\text { institucional }\end{array}$ & $\begin{array}{c}\text { Programa } \\
\text { pedagógico }\end{array}$ & $\begin{array}{c}\text { Textos (frases, } \\
\text { citas breves, } \\
\text { etc.) - } \\
\text { Observaciones }\end{array}$ \\
\hline $\begin{array}{l}\text { Sábado, } 15 \text { de } \\
\text { febrero de } \\
1908 \\
\text { Visita a la } \\
\text { Escuela } \\
\text { Normal de } \\
\text { Bruselas y la } \\
\text { escuela } \\
\text { modelo } \\
\text { M. Alexis } \\
\text { Sluys (1849- } \\
\text { 1936) }\end{array}$ & 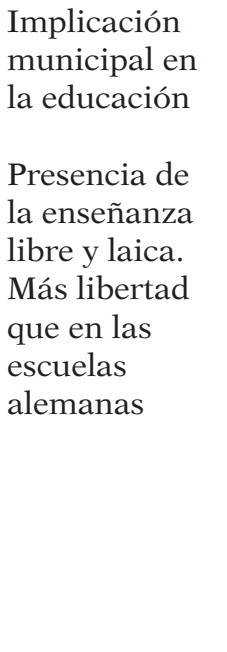 & $\begin{array}{l}\text { Importancia } \\
\text { de la dirección } \\
\text { pedagógica de } \\
\text { M.Sluys en la } \\
\text { escuela } \\
\text { modelo de } \\
\text { Bruselas }\end{array}$ & $\begin{array}{l}\text { Clase de } \\
\text { gimnasia. } \\
\text { Clase de } \\
\text { aritmética. } \\
\text { Clase de } \\
\text { ciencias } \\
\text { naturales: } \\
\text { lección sobre } \\
\text { el origen de } \\
\text { los } \\
\text { manantiales } \\
\text { con el uso de } \\
\text { arcilla y arena } \\
\text { para realizar } \\
\text { experimentos } \\
\text { sencillos }\end{array}$ & $\begin{array}{l}\text { "Vaig sentir } \\
\text { allí la lliçó } \\
\text { més bonica } \\
\text { que jo hagi } \\
\text { sentit donar } \\
\text { als nois en } \\
\text { tota una vida. } \\
\text { D’una } \\
\text { senzillesa } \\
\text { encantadora i } \\
\text { una concisió i } \\
\text { exactitud } \\
\text { admirables, } \\
\text { donada amb } \\
\text { un rigorós } \\
\text { mètode } \\
\text { d'inducció» }\end{array}$ \\
\hline
\end{tabular}

Fuente: elaboración propia. 
Cuadro 2. Síntesis y análisis conceptual

\begin{tabular}{|c|c|c|c|}
\hline $\begin{array}{c}\text { Día/ Mes/ Año } \\
\text { - Lugar - Personas e } \\
\text { instituciones }\end{array}$ & $\begin{array}{l}\text { Elementos del } \\
\text { contexto externo }\end{array}$ & $\begin{array}{c}\text { Contexto } \\
\text { institucional }\end{array}$ & $\begin{array}{c}\text { Programa } \\
\text { pedagógico }\end{array}$ \\
\hline $\begin{array}{l}\text { Viaje pedagógico } \\
\text { de } 1908 \\
\text { Escuela Normal de } \\
\text { Bruselas y la } \\
\text { escuela modelo } \\
\text { M. Alexis Sluys } \\
\text { (1849-1936) }\end{array}$ & $\begin{array}{l}\text { Política educativa } \\
\text { municipal } \\
\text { Enseñanza libre y } \\
\text { laica. } \\
\text { Libertad de } \\
\text { enseñanza y } \\
\text { organización } \\
\text { Escuela modelo }\end{array}$ & $\begin{array}{l}\text { Dirección } \\
\text { pedagógica } \\
\text { Formación del } \\
\text { profesorado }\end{array}$ & $\begin{array}{l}\text { Material de } \\
\text { enseñanza } \\
\text { Método inductivo } \\
\text { Experimentación } \\
\text { Concisión, } \\
\text { exactitud y rigor } \\
\text { explicativo }\end{array}$ \\
\hline
\end{tabular}

Fuente: elaboración propia.

\section{LOS VIAJES EN EL ITINERARIO FORMATIVO Y PROFESIONAL DE ROSA SENSAT}

La biografía personal y profesional de la maestra Rosa Sensat Vilà (1873-1961), que podría sintetizarse con el sintagma «ciencia y renovación de la escuela», ha sido estudiada en profundidad por distintos especialistas. ${ }^{10}$ No repetiremos aquello conocido y estudiado; pero para contextualizar nuestro análisis precisamos situar los viajes pedagógicos de Rosa Sensat, relacionados con los documentos mencionados, entre dos etapas de su biografía. Se trata de la etapa de formación (1883-1913) y la etapa de plenitud profesional (1914-1938). La primera de ellas está constituida por sus estudios, en Barcelona y Madrid, las primeras experiencias profesionales (escuelas de párvulos en Girona y Madrid, la Escuela Normal de Alicante, las escuelas públicas de Sant Martí de Provençals y de la Diagonal en Barcelona) y, como colofón, los viajes pedagógicos de los que nos ocuparemos a continuación. Todo ello constituye la preparación inicial y configura un potente bagaje para acometer su trabajo en la etapa de plenitud profesional con la dirección de la

\footnotetext{
10 Destacamos un trabajo relativamente reciente junto a otro que ya se considera un clásico en el estudio de la obra pedagógica de Rosa Sensat: Albert Esteruelas, Jordi Garcia-Farrero e Isabel Vilafranca, "L'Escola del Bosc cent anys després. Allà on Rosa Sensat va sistematitzar la seva pedagogia», Temps d'Educació 49 (2015): 111-133; Josep González-Agàpito, Rosa Sensat $i$ Vilà, fer de la vida escola (Barcelona: Rosa Sensat/ Edicions 62, 1989).
} 
Escola de Bosc (1914-1931)11 y el Grup Escolar Milà $i$ Fontanals (19311938), así como la presidencia de la sección de Educación del Institut de Cultura i Biblioteca Popular de la Dona a partir del año 1921, los tres centros ubicados en Barcelona. ${ }^{12}$

En este contexto de desarrollo personal y profesional, casi como una culminación de un sólido y completo programa de formación, Rosa Sensat realizó, entre 1908 y 1913, hasta tres viajes de carácter pedagógico que la llevaron a recorrer distintos países europeos en los cuáles visitó instituciones relevantes y conoció pedagogos y responsables educativos de fuerte prestigio en su entorno.

En el año 1908, aprovechando un viaje de negocios de su esposo David Ferrer Vallès, la maestra visitó escuelas de distintas ciudades alemanas (Berlín, Dresde, Leipzig y Colonia), además de Bruselas y París, última etapa del viaje en la que ya no visitó ninguna escuela. El viaje tuvo una duración de tres semanas, entre el 30 de enero y el 21 de febrero. Aparte de las visitas realizadas a escuelas públicas e instituciones educativas de las ciudades alemanas, Rosa Sensat quedó complacida de la estancia en Bruselas, a pesar de su corta duración limitada a dos días. En la ciudad belga conoció a Alexis Sluys (1849-1936), un personaje clave en el proceso de modernización y renovación de la enseñanza belga que fue director de la Escuela modelo y de la Escuela Normal de Bruselas. ${ }^{13}$ En esta ciudad visitó la Escuela Normal, la Escuela modelo y una escuela menagère y profesional.

El viaje del año 1911 fue realizado conjuntamente con un grupo de catorce maestros de las escuelas municipales de Barcelona para visitar escuelas e instituciones educativas de Francia, Suiza y Alemania. El 9 de julio, el grupo de maestros, en el que destacan algunos conocidos nombres propios, estaba formado por Celestina Vigneaux, Rosa

\footnotetext{
${ }^{11}$ Véase: Rosa Sensat Vilà, Hacia la nueva escuela (Madrid: Publicaciones de la Revista de Pedagogía, 1934).

12 Véase: Rosa Sensat Vilà, Discurs de l'acte inaugural del curs 1922-1923 (Barcelona: Institut de Cultura i Biblioteca Popular de la Dona, 1922).

13 Véase: Alexis Sluys, Mémoires d'un pédagogue (Bruxelles, Éditions de la Ligue de l’Enseignement et de l'Éducation Permanente asbl, 2014); George Laurent, "Alexis Sluys: un pédagogue engagé au service de l'enseignement officiel en Belgique», Cahiers Bruxellois - Brusselse Cahiers XLVI, no.1 (2015): 74-106. URL: https://www.cairn.info/revue-cahiers-bruxellois-2015-1-page-74.htm
} 
Sensat, Dolors Cortés, Blanca Martín, Àngels Muncunill, Maria Carbonell, Mercè Padrós, Fernando Gordillo, Basilio Jiménez, Vicente Pinedo, Blas Vernet, Esteban Isern, Llorenç Jou y Josep Udina. Emprendieron un itinerario que les llevó a las ciudades de Tarascón, Lyon, Ginebra, Lausana, Berna, Lucerna, Zurich, Estrasburgo, París y Toulouse, llegando de nuevo a Barcelona el día 28 del mismo mes. Los objetivos están plasmados en la publicación ya citada de la memoria del viaje:

Salimos, por fin, catorce, siete maestros y siete maestras, en el expreso de Francia del día 9 de julio, teniendo la satisfacción de ser despedidos por un buen número de compañeros, jefes y autoridades, y ya en el tren, nos distribuimos el trabajo para que éste resultase aprovechable. No era posible que en tan pocos días (diez y nueve), haciendo un recorrido tan largo, disponiendo de tan corto tiempo para cada población, pudiera hacerse detenido estudio sin que cada cual tuviera su campo de operación concreto; así es que unos se encargaron de los edificios, otros del material de enseñanza, otros del mobiliario, otros de programas, otros de métodos y sistemas, otros del profesorado, otros de leyes y reglamentos, otros de trabajos manuales, otros de enseñanza privada, otros de obras post y circumescolares, etc., y de este modo cada uno tomaría las notas de su respectiva obligación, y el conjunto sería, si no cosa acabada, por lo menos lo más completa en relatividad. ${ }^{14}$

Entre el 15 de octubre de 1912 y el 17 de marzo de 1913, Rosa Sensat realizó el más completo de los tres viajes. Se trata de un viaje de estudios, pensionada por la Junta para la Ampliación de Estudios, con estancias más duraderas y visitando escuelas e instituciones educativas de Bélgica, Suiza y Alemania. No cabe duda de la importancia de este viaje pedagógico en la formación de Rosa Sensat, habida cuenta, de manera específica, de la etapa dedicada a la estancia en Bruselas dónde tuvo estrecha relación con Ovide Decroly en su escuela de la calle l'Hermitage:

\footnotetext{
14 Vigneaux et al, Viaje pedagógico a Francia, Suiza y Alemania en 1914, 4.
} 
A Bèlgica assisteixo a les conferències del doctor Decroly. Les seves idees sobre l'educació nova es troben en plena difusió. Les seves investigacions a l'escola de la rue de l'Ermitage són notabilíssimes en aquest primer període i s'ofereixen amb generositat a la consideració dels mestres estrangers. Les aules, arsenal d'objectes aportats pels alumnes, són una mostra evident que allí regna un esperit d'investigació i d'estudi i que l'ensenyament és viu i real. L'espectacle d'aquelles taules plenes de minerals, de petxines, de retalls de diaris, de dibuixos i d'estampes; aquells prestatges plens d'aquàriums i terraris; aquells infants que treballaven lliurement enmig d'aquell material viu, que era la natura mateixa posada al seu abast, portada a l'escola per les pròpies mans i pel seu mateix afany de recerca i de descoberta, van influir profundament en el meu esperit i em van suggerir, sense cap mena de dubte, moltes de les inspiracions futures. ${ }^{15}$

El interés de Rosa Sensat en este viaje de 1912-1913 se centraba en la didáctica de las ciencias físico-químicas y naturales tal como ella misma manifestó en la solicitud dirigida a la JAE. ${ }^{16}$ Ello determinó, como veremos más adelante en el caso de Ginebra, gran parte de las valoraciones de Rosa Sensat sobre los lugares e instituciones visitadas.

Pedro Luis Moreno, en un documentado y riguroso artículo de análisis de las concepciones de Rosa Sensat sobre el material de enseñanza, dedica un apartado a los viajes pedagógicos y ratifica nuestra apreciación sobre la importancia de los viajes, y éste último de manera especial, en la formación de la maestra:

Els viatges pedagògics li van permetre reforçar i ampliar la seva formació, li van possibilitar l'oportunitat d'observar i conèixer personalment la plasmació d'algunes de les iniciatives més destacades del moviment internacional de l'Escola Nova. L'experiència viatgera li facilitaria un coneixement més profund

\footnotetext{
15 Rosa Sensat, Vers l'escola nova (Vic: Eumo, 1996): 6-7. Traducción al catalán de: Hacia la nueva escuela (Madrid: Publicaciones de la Revista de Pedagogía, 1934).

16 Véase: Expediente Rosa Sensat i Vilà. URL: http://archivojae.edaddeplata.org/jae_app/
} 
i directe de les idees pedagògiques, la situació d'un ensenyament primari modern perfectament estructurat, així com les pràctiques educatives innovadores impulsades per les corrents de renovació pedagògica europees. ${ }^{17}$

\section{GINEBRA: UNA ESCALA OBLIGADA ENTRE BRUSELAS Y LAUSANA}

«Después de haber estudiado a fondo la labor escolar de Bruselas, la escuela primaria de Suiza nos produjo decepción. Ni en su modo de hacer ni en el espíritu que la informa está a la altura de la plenitud que los tiempos demandan». ${ }^{18}$ La cita de Rosa Sensat denota una sensación de decepción con relación al contacto directo con las instituciones educativas de Ginebra, sobre todo en el caso de las escuelas de enseñanza primaria. Sin embargo, en sus obras posteriores se definió ella misma como fruto de la moderna pedagogía desarrollada en algunas de las instituciones visitadas y, más particularmente, bajo la influencia de psicólogos de la talla de Claparède. ${ }^{19}$

La maestra visitó esta ciudad, en las orillas del lago Léman, en dos ocasiones: el viaje en grupo de 1911 y el viaje de estudios de 19121913. La lectura y análisis de los respectivos diarios y memorias nos permite presentar el cuadro 3 en el cual damos cuenta de los centros visitados.

\footnotetext{
${ }_{17}$ Pedro Luis Moreno Martínez, «Rosa Sensat, la cultura material de l'escola i el material d'ensenyament», Temps d'Educació 44 (2013): 87.

18 Sensat, Borrador de la memoria Métodos para la enseñanza de las ciencias físico-naturales en la escuela primaria (Viaje 1912-1913).

19 Esta explícita autoafiliación en la psicología y pedagogía de Claparède contrasta con algunas afirmaciones de sus diarios. Sirva como ejemplo la anotación escrita el viernes 10 de enero de 1913: "Claparède es de los sabios que creen que no han de esforzarse para hacerse comprender de los demás. Será muy sabio, pero dice las cosas a medio decir, sin ningún relieve ni calor, es decir sin las condiciones de una lección dada por un buen maestro».
} 
Cuadro 3. Instituciones ginebrinas visitadas en 1911 y en 1913

\begin{tabular}{|c|c|}
\hline Viaje de 1911 & Viaje de 1913 \\
\hline $\begin{array}{l}\text { Llegada: } 12 \text { de julio } \\
\text { Salida: } 14 \text { de julio en dirección a } \\
\text { Lausana } \\
\text { Jornada completa de visitas: } 13 \text { de julio } \\
\text { Instituciones visitadas } \\
\text { - Collège Calvin (secundaria) } \\
\text { - Institut de jeunes filles } \\
\text { - École primaire des Cropettes } \\
\text { - École professionelle de jeunes gens } \\
\text { - École professionelle et menagère de } \\
\text { - jeunes filles } \\
\text { - École de la petite enfance } \\
\text { - Département d'Instruction publique }\end{array}$ & $\begin{array}{l}\text { Llegada: } 9 \text { de enero } \\
\text { Salida: } 23 \text { de enero hacia Lausana } \\
\text { Instituciones visitadas } \\
\text { - Instituto J. J. Rousseau } \\
\text { - Escuelas de primaria } \\
\text { - École de Malagnou } \\
\text { - École de Roserai } \\
\text { - École de Paquis } \\
\text { - École de Sécheron } \\
\text { - Collège Calvin (secundaria) } \\
\text { - Institut de jeunes filles } \\
\text { - Escuela infantil de Malagnou } \\
\text { - Escuelas particulares } \\
\text { - Escuela "menagère" de Carouge } \\
\text { - Museo de Historia Natural } \\
\text { - Jardín Botánico }\end{array}$ \\
\hline
\end{tabular}

Fuente: elaboración propia.

En los años que nos ocupan, Ginebra se había convertido en un centro de atracción para los maestros y pedagogos catalanes, sobre todo a partir de la apertura, en 1912, del Instituto Jean-Jacques Rousseau (IJJR). La relativa proximidad, en comparación a otros destinos europeos de igual o más prestigio, pero más alejados, confirió a esta ciudad suiza un carácter especial. Ello se debió también, sin ninguna duda, a la lengua de uso más habitual en Ginebra, el francés, de dominio más extendido entre los maestros catalanes, lo cual facilitaba la comunicación y el aprovechamiento de la estancia en la ciudad. El carácter abierto, tolerante e internacionalista de la ciudad reforzaba este papel de destino buscado para experimentar la convivencia entre religiones distintas, la coexistencia de una clara diversidad lingüística y, en fin, la posibilidad de vivir en un ambiente liberal y democrático conocido como el espíritu de Ginebra.

La ciudad se convirtió, también gracias al papel relevante de Ferrière en la creación y desarrollo de la Liga Internacional de la Nueva Educación, 
en una concreción de la utopía político-pedagógica del primer tercio del siglo XX que conectó perfectamente con las aspiraciones y preocupaciones de la burguesía catalana, en primera instancia, y de los sectores progresistas y republicanos, con posterioridad. Ambos perseguían el objetivo de construir un país a través de la educación. ${ }^{20}$

Esta significación especial y, en cierto modo, paradigmática de Ginebra nos ha llevado a tomar la decisión de presentar con más detenimiento y profundidad el contenido pedagógico asociado a la estancia en esta ciudad, aunque lo mismo podría realizarse con Bruselas y Lausana.

Si en el viaje de 1911 la estancia en Ginebra se limitó a un día completo de visitas, durante el mes de enero de 1913, Rosa Sensat permaneció en la ciudad durante dos semanas y, gracias a los buenos oficios del también maestro catalán Pau Vila, que cursaba estudios en el Instituto Jean-Jacques Rousseau y conocía bien la ciudad y sus instituciones, aprovechó con intensidad cada día y cada hora. Cabe recordar que Vila fue el primer estudiante que se matriculó en este nuevo instituto pedagógico ginebrino creado por Claparède y dirigido por Bovet desde 1912, o sea solamente unos meses antes.

Ya hemos señalado anteriormente que, en esta ocasión, el objetivo nuclear del viaje de Rosa Sensat era la enseñanza de las ciencias. Ello influye en su visión y en la atención que presta a otros temas. Sin embargo, este explícito y concreto interés no fue motivo suficiente para que la maestra no abriera el foco de sus motivaciones y ampliase el horizonte de sus preocupaciones. El siguiente cuadro es una demostración de la afirmación que acabamos de formular. La síntesis de los elementos conceptuales que aparecen en los diarios de los dos viajes nos ofrece una visión de la amplitud y la extensión del universo conceptual que se estaba tejiendo en la construcción del pensamiento pedagógico de la maestra, sistematizado a partir del esquema de análisis que hemos justificado anteriormente.

\footnotetext{
${ }^{20}$ Véase: Joan Soler Mata, «Influència i presència de l'Institut Rousseau de Ginebra en la pedagogia catalana del primer terç del segle XX», Temps d'Educació 37 (2009): 11-38.
} 
Cuadro 4. Síntesis y análisis conceptual de las estancias en Ginebra

\begin{tabular}{|c|c|c|}
\hline $\begin{array}{l}\text { Elementos del contexto } \\
\text { externo }\end{array}$ & Contexto institucional & Programa pedagógico \\
\hline $\begin{array}{l}\text { Política educativa } \\
\text { municipal y cantonal } \\
\text { Enseñanza "menagère" } \\
\text { Enseñanza de párvulos } \\
\text { Formación de maestros } \\
\text { Escuela pública } \\
\text { Escuela libre (particular) } \\
\text { Gratuidad y obligatoriedad } \\
\text { Experimentación y } \\
\text { extensión de los resultados } \\
\text { (generalización) }\end{array}$ & $\begin{array}{l}\text { Edificios y mobiliario } \\
\text { Condiciones higiénicas } \\
\text { Modelo de pupitre } \\
\text { Dirección pedagógica } \\
\text { Graduación escolar } \\
\text { Maestro: función de } \\
\text { enseñanza } \\
\text { Laicismo y tolerancia } \\
\text { Educación especial en los } \\
\text { centros (clase de } \\
\text { anormales y clase de } \\
\text { atrasados) } \\
\text { Escuela a la medida } \\
\text { Escuela como la vida }\end{array}$ & $\begin{array}{l}\text { Fundamentos psicológicos } \\
\text { y pedagógicos } \\
\text { Educación integral } \\
\text { Libertad del niño } \\
\text { Lecciones de cosas } \\
\text { Enseñanza memorística } \\
\text { Método experimental } \\
\text { Método inductivo } \\
\text { Educación individualizada } \\
\text { Música y gimnasia } \\
\text { Dibujo: base de la } \\
\text { enseñanza (maestro) y del } \\
\text { trabajo manual (alumno) } \\
\text { Materiales de enseñanza } \\
\text { Decoración del aula }\end{array}$ \\
\hline
\end{tabular}

Fuente: elaboración propia.

Rosa Sensat se interesó con más intensidad por aquello que configuraba el carácter de la escuela; es decir, por su contexto institucional y por el programa pedagógico. En las descripciones y reflexiones de sus visitas se ocupa de analizar todo lo relacionado con la escuela como institución, desde el edificio y mobiliario (en particular sobre las condiciones higiénicas y los modelos de pupitre), hasta el carácter de la escuela, el tipo de dirección pedagógica de los centros (y, a veces, la ausencia de este tipo de dirección que para ella era indispensable), la función de enseñanza en el maestro y, en último término, la concepción de escuela que está asociada a un determinado establecimiento escolar (la escuela a la medida, la escuela como la vida, etc.). Es evidente que no escaparon a las apreciaciones de la maestra algunos elementos de especial relevancia en relación con la aplicación posterior de sus estudios. Se trata de elementos del contexto externo o de política educativa, como el papel del municipio y el cantón en la organización de la enseñanza, la gratuidad y obligatoriedad de la misma y, en algunos casos, los procesos a través de los cuales la experimentación realizada en una escuela se traducía en la extensión y la posible generalización de resultados a otros centros. Sin 
duda, Rosa Sensat tenía en mente, a cada momento, aquello que formaría parte de su vida profesional posterior en Barcelona, una ciudad que vivía el impulso de la iniciativa municipal en la educación y que se debatía entre la necesidad de experimentar sin dejar de generalizar para conseguir una mejora global de las escuelas de la ciudad.

El cuadro anterior también deja constancia del interés de Rosa Sensat por el programa pedagógico, o sea por aquello que ocurría en el interior del aula y por la manera de llevar a cabo la enseñanza, más concretamente la enseñanza de las ciencias. En este apartado su interés iba desde los fundamentos y las concepciones educativas, hasta el tipo de lecciones y las metodologías aplicadas, e incluso los materiales y el ambiente del aula. Cabe destacar la importancia que daba al dibujo que ella concebía como un elemento básico para que el maestro pudiera enseñar y, a la vez, para desarrollar las aptitudes del trabajo manual en los alumnos.

La interpretación de estos elementos conceptuales, a partir de los textos de Rosa Sensat, nos permite dar un paso más y establecer un cuadro en el que reflejamos los ejes conceptuales que emergen de la reflexión pedagógica de la maestra. Se trata de unos ejes conceptuales que dan buena cuenta de la amplitud de sus preocupaciones, sin abandonar nunca la capacidad de focalizar en los temas relacionados más directamente con sus intereses. A la vez, estos ejes constituyen temas de debate que dibujan los trazos imprescindibles para comprender el verdadero núcleo del programa renovador que Rosa Sensat estaba construyendo.

Cuadro 5. Ejes conceptuales - Temas de debate

\begin{tabular}{|c|c|c|}
\hline Contexto externo & Contexto institucional & Práctica escolar \\
\hline $\begin{array}{l}\text { Educación pública } \\
\text { Generalización - } \\
\text { Institucionalización de la } \\
\text { renovación } \\
\text { Responsabilidad de la } \\
\text { política educativa } \\
\text { Teoría - Práctica }\end{array}$ & $\begin{array}{l}\text { Graduación } \\
\text { Dirección del centro } \\
\text { Laicismo y neutralidad } \\
\text { Participación del alumno } \\
\text { Escuela a la medida } \\
\text { Coeducación. Separación } \\
\text { por sexo } \\
\text { Separación por niveles }\end{array}$ & $\begin{array}{l}\text { Libertad del niño: clase } \\
\text { dirigida - clase libre } \\
\text { Métodos de enseñanza: } \\
\text { coherencia - diversidad } \\
\text { Salidas y excursiones: } \\
\text { oportunidad de } \\
\text { aprendizaje } \\
\text { Experimentación en el } \\
\text { laboratorio, en el aula y en } \\
\text { el patio o campo escolar }\end{array}$ \\
\hline
\end{tabular}

Fuente: elaboración propia. 


\section{CONCLUSIONES}

No cabe duda de que los diarios y las notas de viaje estudiados superan el marco de un diario de viaje y añaden, a sus características, las propias de un diario de investigación, de reflexión crítica e, incluso, de formación. Se trata de un completo material que permite distintas lecturas, algunas de las cuales ya han sido realizadas, como hemos citado anteriormente, por otros investigadores. ${ }^{21}$

La reconstrucción y el análisis del universo conceptual de Rosa Sensat nos permiten establecer confluencias y divergencias entre los tres relatos, localizar temas de debate y sugerir el establecimiento de continuidades entre estudios realizados, visitas y observaciones y el ideario o discurso pedagógico.

En un trabajo anterior, que ya hemos citado, establecimos seis posibilidades para comprender lo que los maestros catalanes buscaron y encontraron en Ginebra:22

- La oportunidad de establecer y fortalecer vínculos con la Liga Internacional de la Nueva Educación.

- La motivación de quienes descubrieron en Ginebra nuevos itinerarios pedagógicos.

- El descubrimiento de la importancia de construir los fundamentos científicos de la educación basada en las enseñanzas de los profesores del IJJR de Ginebra.

- La oportunidad de aprovechar el prestigio del IJJR y la Universidad de Ginebra para fortalecer su propio prestigio personal y profesional.

- La actitud de aquellos que estaban imbuidos de la atmósfera liberal, abierta, pacifista, democrática e internacionalista de Ginebra.

- La constatación que no todo se encontraba en Ginebra.

\footnotetext{
${ }^{21}$ Moreno Martínez, «Rosa Sensat, la cultura material de l'escola i el material d'ensenyament».

22 Soler Mata, «Influència i presència de l'Institut Rousseau de Ginebra en la pedagogia catalana del primer terç del segle XX».
} 
En el caso de Rosa Sensat que nos ocupa en este artículo, podemos deducir que sus viajes estuvieron marcados por la importancia de construir los fundamentos científicos de la educación basados en las enseñanzas de los profesores del Instituto Jean-Jacques Rousseau, aprovechando el prestigio personal y profesional que se obtenía como consecuencia del prestigio de los profesores del Instituto. Sin embargo, es evidente que en Rosa Sensat cabe remarcar la constatación que no todo se encontraba en Ginebra: «Dentro de unos días salgo de Ginebra para Lausanne pues veo que aquí no podría hacer gran cosa sobre la especialidad que he venido a estudiar».

En Ginebra, Rosa Sensat había establecido contacto personal con Pierre Bovet, el director del Instituto Rousseau, Édouard Claparède, con quién asistió a clase en una ocasión, Alice Descoeudres, la encargada del curso sobre alumnos discapacitados en el mismo instituto, la inspectora de gimnasia Mme. Ballet, la pintora y profesora de dibujo Mme. Louise Artus-Perrelet ${ }^{23}$ y, finalmente, con Mme. Deletré, la directora de un colegio particular. En ningún caso la intensidad de la relación puede compararse con la establecida en Bruselas con Decroly y Sluys. Ello se desprende de la descripción y los comentarios de Rosa Sensat en sus diarios y anotaciones.

A pesar de la relativa importancia de los aprendizajes realizados durante su estancia en Ginebra podemos señalar algunos elementos clave de su ideario pedagógico que tienen una clara filiación en la pedagogía ginebrina. Se trata de elementos que ella misma aporta como síntesis de su viaje y en los cuáles hemos señalado en negrita aquellos que tienen claros referentes ginebrinos, aunque no sea de forma exclusiva, a partir de lo leído e interpretado de sus diarios:

- El municipio y el ayuntamiento como ámbito de la política escolar.

- La escuela como buen-lugar: edificio, ambiente y condiciones higiénicas; laboratorios y campos de experimentación.

- La organización de la escuela como base de la enseñanza: graduación y dirección pedagógica.

${ }^{23}$ Louise Artus-Perrelet, Le Déssin au service de l'éducation (Neuchâtel: Delachaux et Niestlé, 1917). 
- La función educativa antes que la instructiva: el trabajo individual.

- La necesidad de formación del maestro: carácter técnico y profesional; fundamentos teóricos y recursos metodológicos.

- La importancia del material de enseñanza en el aula y en la escuela.

- El entorno natural, social y cultural como fuente de conocimiento.

- El principio de la actividad del niño: la importancia del trabajo manual.

- La relación entre la escuela y la vida: enseñanza de las ciencias y vida doméstica.

- La formación específica de la mujer.

En el diagrama que sigue a continuación hemos representado los ejes principales de un balance del modelo pedagógico de Rosa Sensat en relación con los temas y conceptos que hemos analizado a lo largo del artículo. Sin ninguna pretensión de limitar el modelo, intentamos establecer cinco ámbitos temáticos que, a nuestro entender y a partir del análisis de los textos reseñados, constituyen el entramado de un edificio pedagógico que, aunque ya contaba con puntos de anclaje, se robusteció durante los viajes y terminó definiéndose en la práctica educativa que siguió en la Escola de Bosc de Barcelona. 
Gráfico 2. El modelo pedagógico de Rosa Sensat

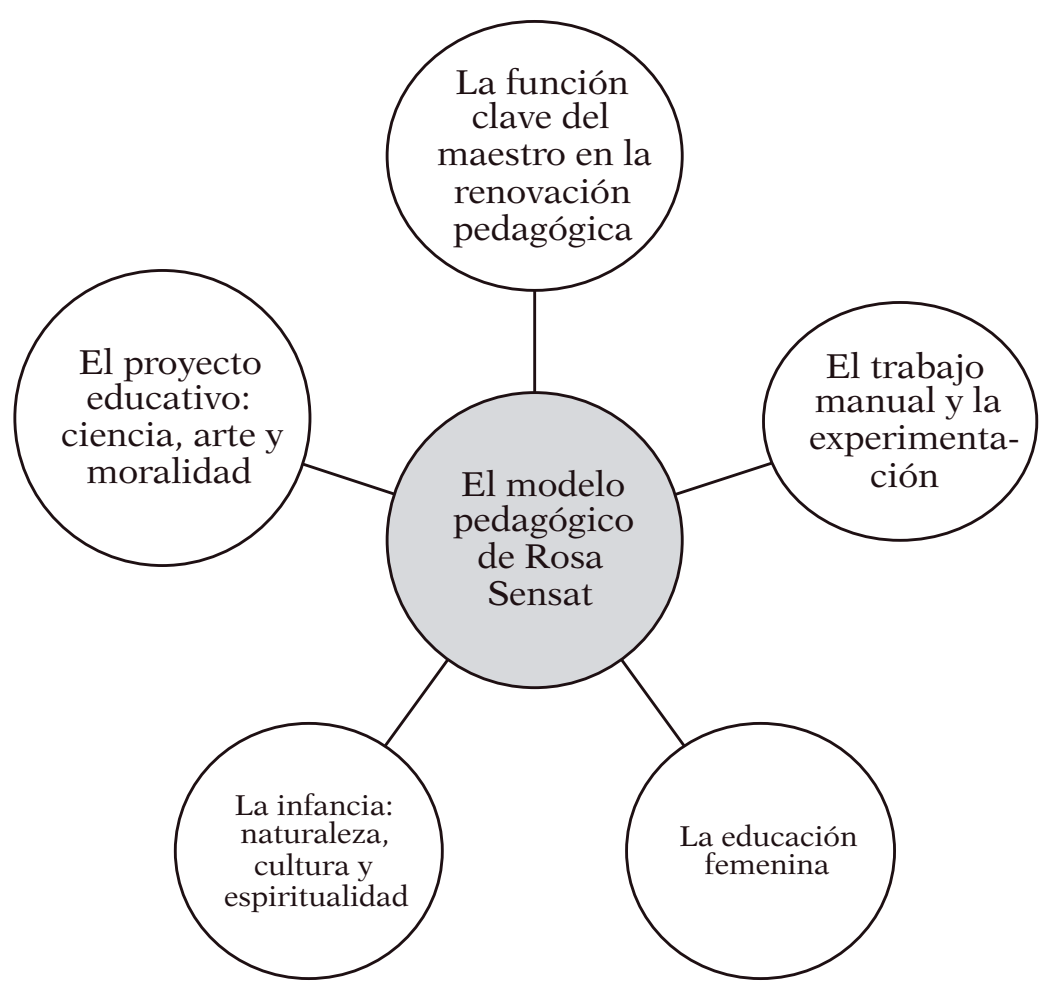

Fuente: elaboración propia.

Es interesante descubrir cómo el objetivo inicial centrado en el interés y el estudio de las cuestiones metodológicas y didácticas derivadas de la enseñanza de las ciencias físico-químicas y naturales queda desbordado por un marco más amplio que se adentra en múltiples y complejas cuestiones de carácter psicológico y pedagógico. Estas cuestiones, que sirven perfectamente como conclusiones, se intentan sintetizar y sistematizar en forma de recomendaciones redactadas por la misma Rosa Sensat en la parte final del borrador de la memoria del viaje de 1912-1913: edificios escolares espaciosos e higiénicos con campos circundantes y de experimentación; organización y graduación de la enseñanza; incorporación del trabajo manual «seriamente organizado»; estudio de las ciencias naturales; reforma de las Escuelas Normales «acentuando el carácter técnico y profesional» de la formación de los 
maestros; formación permanente de los maestros en forma de cursos de vacaciones; obligatoriedad de las excursiones escolares y su metodología y organización; impulso de las escuelas secundarias y superiores dirigidas a la mujer; y enseñanza de la economía doméstica fundamentada en las ciencias. ${ }^{24}$

\section{Nota sobre el autor}

JoAn Soler Mata es maestro y pedagogo. Doctor en Pedagogía por la Universidad de Barcelona. Actualmente es profesor del Departamento de Pedagogía de la UVIC-UCC. Ha ocupado distintos cargos de gestión universitaria: director del Departamento de Pedagogía; coordinador de los estudios de Maestro; adjunto del Vicerrectorado de Ordenación académica; responsable de la planificación de los trabajos de final de Grado y, también, decano de la facultad.

Es miembro del Grup de Recerca Educativa de la Universitat de Vic (GREUV) — grupo de investigación consolidado-, desde su creación. Actualmente (y desde el año 2015), es presidente de la Societat d'Història de l'Educació dels Països de Llengua Catalana. Los ámbitos de estudio e investigación son: la formación del maestro y del educador social; la historia de la renovación pedagógica en Cataluña; el pensamiento educativo contemporáneo; las relaciones entre territorio, municipio y educación; la escuela rural; la democracia en la escuela. Ha publicado artículos y capítulos de libro sobre los ámbitos de estudio e investigación señalados. También ha coordinado y editado libros de distintos autores sobre la renovación pedagógica y la pedagogía contemporánea.

Actualmente es coordinador del grupo de trabajo de «Lliçons de Pedagogia», un proyecto de innovación en el ámbito de la enseñanza de la historia de la educación, impulsado por el programa MIF para la mejora de la formación inicial de los maestros de Catalunya.

\footnotetext{
${ }^{24}$ Véase el cuaderno 28 de Sensat, Borrador de la memoria Métodos para la enseñanza de las ciencias físico-naturales en la escuela primaria (Viaje 1912-1913).
} 


\section{REFERENCIAS}

Artus-Perrelet, Louise. Le Désin au service de l'éducation. Neuchâtel: Delachaux et Niestlé, 1917.

Bolívar, Antonio, Jesús Domingo y Manuel Fernández. La investigación biográfico-narrativa en educación. Enfoque y metodología. Madrid: La Muralla, 2001.

Esteruelas, Albert, Jordi Garcia-Farrero e Isabel Vilafranca. «L'Escola del Bosc cent anys després. Allà on Rosa Sensat va sistematitzar la seva pedagogia». Temps d'Educació 49 (2015): 111-133.

González-Agàpito, Josep. Rosa Sensat $i$ Vilà, fer de la vida escola. Barcelona: Rosa Sensat/ Edicions 62, 1989.

Laurent, George. «Alexis Sluys: un pédagogue engagé au service de l'enseignement officiel en Belgique». Cahiers Bruxellois - Brusselse Cahiers XLVI, no. 1 (2015): 74-106. URL: https://www.cairn.info/revue-cahiers-bruxellois-2015-1-page-74. htm

Moreno Martínez, Pedro L. «Rosa Sensat, la cultura material de l'escola i el material d'ensenyament». Temps d'Educació 44 (2013): 77-99.

Oncina Coves, Faustino. «Historia conceptual y hermenéutica». Azafea. Revista de Filosofía 5 (2003): 161-190.

Oncina Coves, Faustino (ed.). Palabras, conceptos, ideas. Estudios sobre la historia conceptual. Barcelona: Herder, 2010.

Ricoeur, Paul. La memoria, la historia y el olvido. Madrid: Trotta, 2003.

Sensat Vilà, Rosa. Borrador de la memoria Métodos para la enseñanza de las ciencias físico-naturales en la escuela primaria (Viaje 1912-1913). Fondo Rosa Sensat. Archivo Histórico de la Biblioteca Rosa Sensat de Barcelona. Disponible en: http://www2.rosasensat.org/es/pagina/fons-historic

Sensat Vilà, Rosa. Discurs de l'acte inaugural del curs 1922-1923. Barcelona: Institut de Cultura i Biblioteca Popular de la Dona, 1922.

Sensat Vilà, Rosa. Vers l'escola nova. Vic: Eumo, 1996. Traducción al catalán de: Hacia la nueva escuela. Madrid: Publicaciones de la Revista de Pedagogía, 1934.

Sluys, Alexis. Mémoires d'un pédagogue. Bruxelles: Éditions de la Ligue de l'Enseignement et de l'Éducation Permanente asbl, 2014.

Soler Mata, Joan. «Influència i presència de l’Institut Rousseau de Ginebra en la pedagogia catalana del primer terç del segle XX». Temps d'Educació 37 (2009): 11-38.

Vigneaux, Celestina et al. Viaje pedagógico a Francia, Suiza y Alemania en 1911. Memoria presentada al Ayuntamiento de Barcelona por varios Maestros públicos de dicha ciudad. Barcelona: J. Horta impresor, 1912.

Vilanou, Conrad. «Historia conceptual e Historia de la educación». Historia de la Educación 25 (2006): 35-70. 
Vilanou, Conrad y Joan Soler. «La Historia de la Educación en Cataluña: tradición liberal e historia conceptual». Social and Education History 2, no. 3 (2013): 261-295. URL: http://dialnet.unirioja.es/descarga/articulo/4686204. pdf

Viñao, Antonio. «Las autobiografías, memorias y diarios como fuente histórico-educativa: tipología y usos». Sarmiento. Anuario Galego de Historia da Educación 3 (1999): 223-253. 